Gut, 1976, 17, 68-74

\title{
Limitations of ultracentrifugation and in vivo dialysis as methods of stool analysis
}

\author{
C. W. I. OWENS AND W. PADOVAN ${ }^{1}$ \\ From the Medical Unit, University College Hospital Medical School, London
}

SUMMARY Extracellular stool fluid has been sampled by in vivo dialysis, in vitro dialysis, and high speed centrifugation. Detailed comparisons of amino acids and electrolytes have been made between each fluid; normal values and significant differences are summarized. Evidence is provided which suggests that there is release of intracellular contents during normal handling of stool in the laboratory. The amino acids which display the greatest differences between centrifugate and dialysate from given stool specimen are those which are liberated by ultrasonication or aerobic incubation of stool. Factors affecting the chemical stability of stool are discussed. In most circumstances in vivo dialysis would seem to provide the closest approximation to extracellular fluid, providing the faeces remain in the descending colon or rectum for at least two hours.

Technical difficulties in purification of faeces for analysis have delayed evaluation of colonic metabolism and the discovery of biochemical details in health and disease.

Wrong et al. (1961) introduced in vivo dialysis which provides fluid that can be analysed by conventional techniques and which is described as being in equilibrium with stool water (Wrong et al., 1965). More recently, Tarlow and Thom (1974) assessed the value of centrifugation, at $40000 \mathrm{~g}$ and $0^{\circ} \mathrm{C}$ for 30 minutes, as a method of obtaining extracellular fluid from homogenized soft stool. Their centrifugate differed significantly from in vivo dialysate in several respects and it was suggested that ingested dialysis bags are not in true equilibrium with stool water.

While developing a method for the quantitative estimation of faecal amino acids, we had the opportunity of further assessing the difference between ultracentrifugate and dialysate (the U-D difference) which we shall describe together with some of the factors that may influence it.

\section{Methods}

Faeces, free from urine, were collected from nine males. The donors had no evidence of gastrointestinal disease, had a regular daily bowel habit,

${ }^{1}$ Present address: Department of Clinical Medicine, Medical School of Ribeirao Preto, S.P., Brazil.

Received for publication 20 October 1975 and were not taking medication. Experiments were initiated less than 10 minutes after specimen collection and dilution, if undertaken, was done by adding an equal weight of physiological saline at room temperature, and homogenizing gently in polyethylene bags, deliberately avoiding sheering forces. Dialysis bags containing dextran (Wrong et al., 1965) were usually retrieved 24 to 36 hours after ingestion. Their contents were removed by needle and syringe and stored in sterile containers at $-10^{\circ} \mathrm{C}$ before analysis. Specimens for centrifugation were packed into 15 or $50 \mathrm{ml}$ polypropylene centrifuge tubes and spun at $2^{\circ} \mathrm{C}$ in an MSE Superspeed 50 or MSE High Speed 18 for two hours at 40000,26000 , or $6000 \mathrm{~g}$. Centrifugates were prepared from whole stool, diluted stool, and sonicated samples of diluted stool.

Sonic disruption of cellular components was achieved in diluted specimens, kept near room temperature with ice, by an MSE Ultrasonic Power Unit ( $9 \mathrm{~mm}$ probe) used for 90 seconds.

Amino acid analysis was executed by automatic cation exchange chromatography, using the method of Owens and Padovan (1975). Sodium and potassium were measured by automated flame photometry based on methods described in Technicon Method N21b II (Mk 4 flame photometer) and chloride by the mercuric thiocyanate-ferric ion colorimetric method described in the same manual.

Stool water was estimated by drying specimens to constant weight in weighed open screw cap bottles at $100^{\circ} \mathrm{C}$, cooling them in a dessicator and reweighing closed from air. 


\section{Results}

DIRECT COMPARISON OF CENTRIFUGATE AND DIALYSATE

Paired samples of faecal dialysate and $24000 \mathrm{~g}$ centrifugate were isolated from 16 undiluted specimens provided by nine subjects. The results of electrolyte and amino acid analysis are shown in Tables 1 and 2 and enable direct comparisons to be made. In Table 1, amino acids have been grouped (I-IV) for descriptive purposes, according to the significance of the U-D difference. Clearly, the various amino acids behave very differently in contrast with the electrolytes which are similarly distributed in both fluids. Table 1 displays normal values with standard deviations for both methods of sampling.

\section{RELATIONSHIP BETWEEN U-D DIFFERENCE} AND INTRACELLULAR CONTENTS

An interpretation to explain the variable amino acid U-D differences is that, during centrifugation, there is release of intracellular contents. If true, the U-D difference in a given individual should be uniformly exaggerated by any process causing release of intracellular contents. By reverse extrapolation, it could then be suggested that the initial differences were caused by the same processes. Ultrasonication of dilute stool was chosen as a satisfactory way of releasing cell contents and Fig. 1 shows a direct

\begin{tabular}{llrll}
\hline Electrolyte & $\begin{array}{l}\text { Ultracentri- } \\
\text { fugate }\end{array}$ & \multicolumn{1}{l}{$\begin{array}{l}\text { In vivo } \\
\text { dialysate }\end{array}$} & $n$ & $P$ \\
\hline Sodium & $31 \cdot 17 \pm 10.55$ & $32.83 \pm 4.35$ & 6 & $>0.05$ \\
Potassium & $96.67 \pm 19.93$ & $102.17 \pm 19.23$ & 6 & $>0.05$ \\
Chloride & $27.17 \pm 8.95$ & $30.00 \pm 7.29$ & 6 & $>0.05$ \\
\hline
\end{tabular}

Table 2 Electrolyte concentrations in ultracentrifugate and in vivo dialysate in six stool specimens from five normal individuals

Values are mean $\pm 1 \mathrm{SD}$, in $\mathrm{mEq} / \mathrm{l}$.

positive relationship between the differences in dialysate and $40000 \mathrm{~g}$ centrifugate plotted against the differences between diluted sonicated and nonsonicated specimens of the same stool. The assumption was made and later verified that dilution in itself did not cause marked effects. In a single individual there was a close relationship (Fig. 1) (correlation coefficient 0.90, $\mathrm{P}<0.001, \mathrm{~N}=16$ ) whereas the collected data from five individuals showed a smaller correlation coefficient of 0.59 $(\mathrm{P}<0.001, \mathrm{~N}=104)$.

\section{DIFFUSION STUDIES}

To determine the diffusion characteristics of the dialysis membrane, studies were made in pure aqueous solution, free from rapid concentration changes and metabolic complications. Pre-soaked distended bags were suspended in a magnetically stirred water bath, at $37^{\circ} \mathrm{C}$, containing $500 \mathrm{ml}$ of a

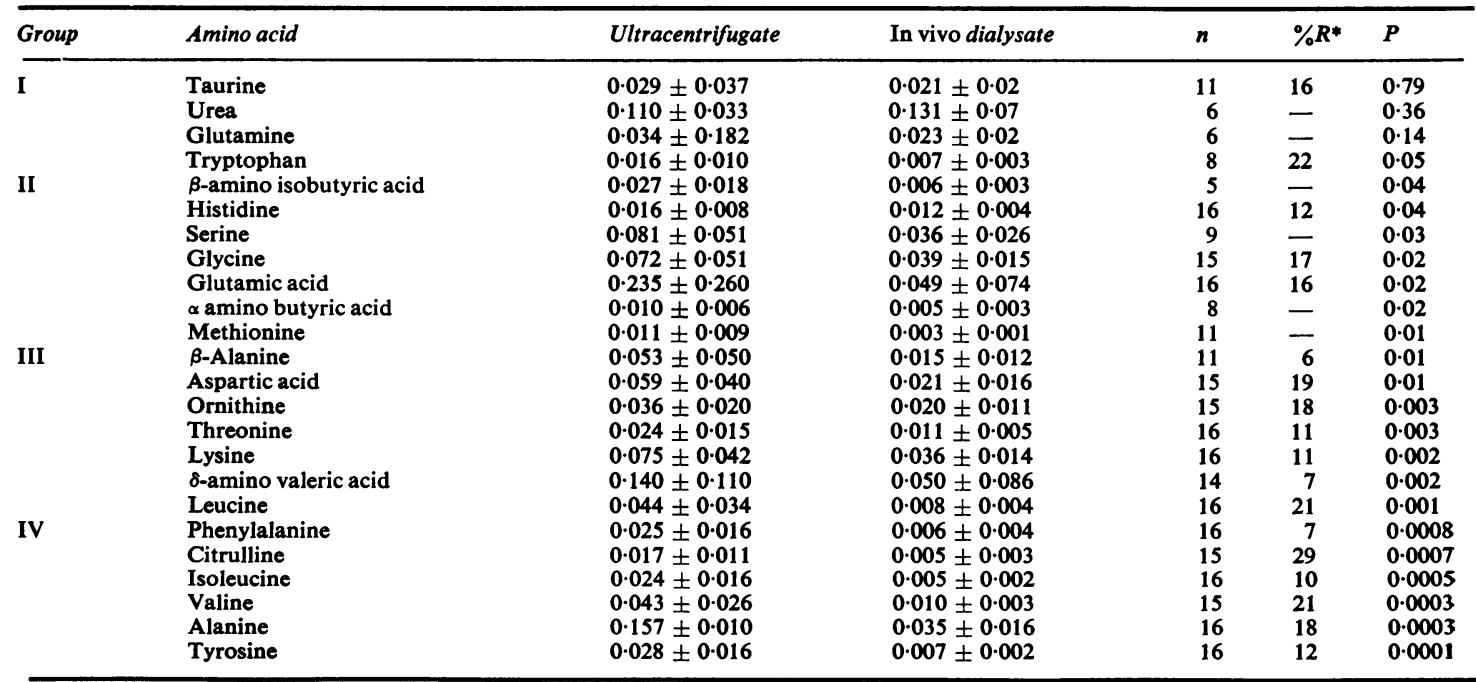

Table 1 Amino acid concentrations in ultracentrifugate and in vivo dialysate obtained simultaneously in 16 stool specimens from nine normal individuals

Values are mean $\pm 1 \mathrm{SD}$, in mmol/1

$* \% \mathbf{R}$ is the reproducibility of the method calculated as the difference of two duplicate estimations divided by their mean multiplied by 100 . Figure quoted is the mean of six duplicate pairs. 


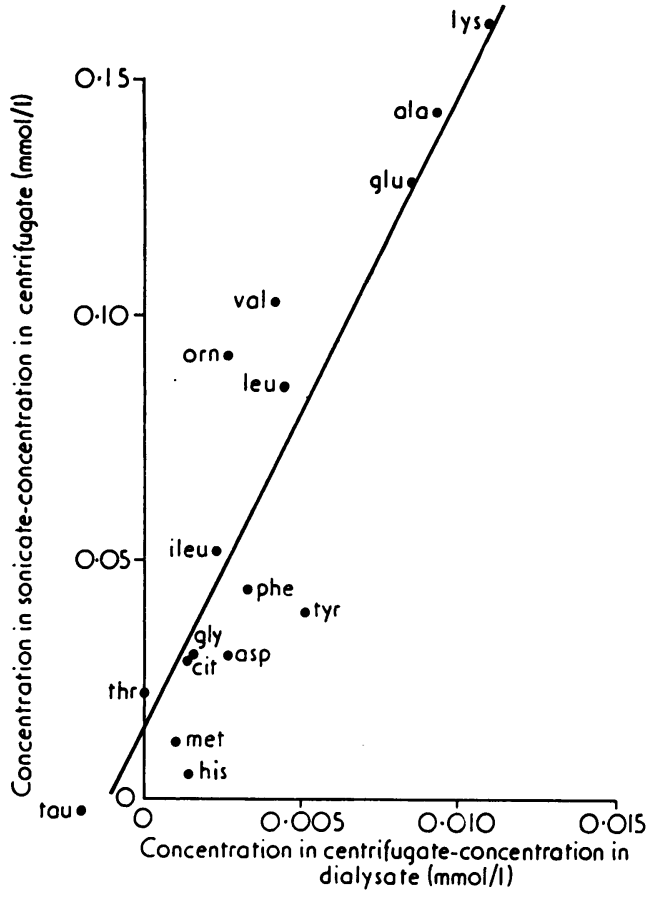

Fig. 1 The relationship of amino-acid concentration changes accompanying centrifugation and sonication.

complex standard amino acid solution $(0.2 \mathrm{mmol} / \mathrm{l})$ and sampled at intervals for four hours. Plotted diffusion curves were of similar shape and showed that most amino acids were more than $90 \%$ equilibrated at 120 minutes (Table 3 ).

To eliminate the possibility that protein or other

\begin{tabular}{|c|c|c|c|}
\hline $\begin{array}{l}\text { Amino } \\
\text { acid }\end{array}$ & $\begin{array}{l}\text { Bags in } \\
\text { aqueous solution }\end{array}$ & $\begin{array}{l}\text { Bags in } \\
\text { ultracentrifugate }\end{array}$ & $\begin{array}{l}\text { Bags in } \\
\text { diluted faeces }\end{array}$ \\
\hline Ure & $94 \cdot 9(35)$ & - & $99 \cdot 5$ \\
\hline Asp & $111 \cdot 1(80)$ & - & $93 \cdot 6$ \\
\hline Thr & $96 \cdot 3(100)$ & $96 \cdot 3$ & $88 \cdot 1$ \\
\hline Ser & $106 \cdot 2(75)$ & $102 \cdot 7$ & 98.5 \\
\hline Gln & - & - & $82 \cdot 6$ \\
\hline Glu & $109 \cdot 5(95)$ & $106 \cdot 7$ & - \\
\hline Cit & - & $86 \cdot 5$ & - \\
\hline Gly & - & $90 \cdot 1$ & $94 \cdot 7$ \\
\hline Ala & $96 \cdot 2(100)$ & $87 \cdot 7$ & 115.9 \\
\hline Val & 90.0 & $99 \cdot 3$ & 112.9 \\
\hline Ile & - & $104 \cdot 6$ & 115.4 \\
\hline Leu & $97 \cdot 7(120)$ & $106 \cdot 6$ & $111 \cdot 6$ \\
\hline Tyr & 90.6 & $95 \cdot 5$ & 103.0 \\
\hline Phe & $94 \cdot 4$ & 96.0 & 95.1 \\
\hline $\mathrm{His}$ & $92 \cdot 7(120)$ & - & 105.5 \\
\hline Orn & $70 \cdot 7(210)$ & $103 \cdot 8$ & $102 \cdot 6$ \\
\hline Lys & $73 \cdot 3(240)$ & $100 \cdot 7$ & $70 \cdot 0$ \\
\hline
\end{tabular}

Table 3 In vitro equilibration of dialysis bags in aqueous amino acid solution, in faecal ultracentrifugate and in diluted faeces

The bag content of each amino acid at two hours is expressed as a percentage of the outside concentration and the $90 \%$ equilibration time in minutes is shown in parentheses. faecal contents may bind to or modify the membrane surface and so introduce artefacts, two presoaked bags were suspended in $40 \mathrm{ml}$ of clear and microscopically cell-free ultracentrifugate at $37^{\circ} \mathrm{C}$. Analysis conducted on the centrifugate initially and on the centrifugate and dialysate after two hours showed that the centrifugate was stable and that within the reproducibility of the analytical method there was good equilibration. With little difference demonstrated between the two compartments in cell-free systems, the situation was investigated in diluted stool. Presoaked bags were immersed in diluted faeces contained in a closed polyethylene bag and maintained at $37^{\circ} \mathrm{C}$, with gentle agitation. A comparison of their contents and a $26000 \mathrm{~g}$ centrifugate of the surrounding fluid was made at two hours and again the results in Table 3 show that, for most substances, there is excellent equilibration, but attention is drawn to relatively higher levels of alanine, valine, isoleucine, and leucine in the surrounding fluid.

ASSESSMENT OF FACTORS INFLUENCING COMPOSITION OF FAECAL CENTRIFUGATE DILUTION

Dilution itself could cause changes in composition and lead to false conclusions in experiments involving this step. To eliminate this possibility $40000 \mathrm{~g}$ centrifugates of whole and diluted portions of the same specimen were compared directly. Results for the critical group IV acids from eight paired estimations failed to reveal significant differences (Table 4), but in general the mean values of the diluted samples were lower. If correction be made for an average water content of $80 \%$ in the original stool, then means for the two groups become very close. We cannot explain the apparent reversal of the situation for isoleucine.

\section{Force of centrifugation}

High speed centrifugation might express intracellular contents, especially from previously damaged cells. Comparison between paired diluted samples centri-

\begin{tabular}{lllll}
\hline $\begin{array}{l}\text { Amino } \\
\text { acid }\end{array}$ & \multicolumn{2}{l}{ Ultracentrifugate } & $n$ & $P$ \\
\cline { 2 - 3 } & Diluted stool & Neat stool & & \\
\hline Phe & $0.022 \pm 0.018$ & $0.027 \pm 0.014$ & 8 & 0.71 \\
Cit & $0.008 \pm 0.002$ & $0.013 \pm 0.009$ & 8 & 0.15 \\
Ile & $0.038 \pm 0.067$ & $0.025 \pm 0.015$ & 8 & 0.91 \\
Val & $0.027 \pm 0.009$ & $0.039 \pm 0.022$ & 7 & 0.20 \\
Ala & $0.099 \pm 0.093$ & $0.150 \pm 0.079$ & 8 & 0.13 \\
Tyr & $0.025 \pm 0.022$ & $0.031 \pm 0.013$ & 8 & 0.71 \\
\hline
\end{tabular}

Table 4 Amino acid concentrations in ultracentrifugates obtained simultaneously from whole and saline diluted stool specimens

Values are mean $\pm 1 \mathrm{SD}$, in $\mathrm{mmol} / \mathrm{l}(\mathrm{N}=8)$. 
fuged at 6000 and $26000 \mathrm{~g}$ showed no significant differences (Table 5). The trend, if any, was in the reverse direction, with higher mean values in the low speed group. Diluted samples from the same individuals centrifuged at $40000 \mathrm{~g}$ had even lower mean values (not shown).

\begin{tabular}{|c|c|c|c|c|}
\hline \multirow{2}{*}{$\begin{array}{l}\text { Amino } \\
\text { acid }\end{array}$} & \multicolumn{2}{|c|}{ Ultracentrifugate } & \multirow[t]{2}{*}{$n$} & \multirow[t]{2}{*}{$\boldsymbol{P}$} \\
\hline & $6000 \mathrm{~g}$ & $26000 \mathrm{~g}$ & & \\
\hline $\begin{array}{l}\text { Glu } \\
\text { Asp } \\
\text { Ala } \\
\text { Gly } \\
\text { Ser } \\
\text { Thr }\end{array}$ & $\begin{array}{l}0.072 \pm 0.040 \\
0.044 \pm 0.016 \\
0.083 \pm 0.026 \\
0.064 \pm 0.041 \\
0.071 \pm 0.037 \\
0.020 \pm 0.007\end{array}$ & $\begin{array}{l}0.017 \pm 0.005 \\
0.022 \pm 0.012 \\
0.048 \pm 0.016 \\
0.039 \pm 0.014 \\
0.053 \pm 0.022 \\
0.019 \pm 0.010\end{array}$ & $\begin{array}{l}4 \\
4 \\
4 \\
4 \\
4 \\
4\end{array}$ & $\begin{array}{l}0.07 \\
0.13 \\
0.18 \\
0.37 \\
0.66 \\
0.99\end{array}$ \\
\hline
\end{tabular}

Table 5 Amino acid concentrations in ultracentrifugates prepared at $6000 \mathrm{~g}$ and $26000 \mathrm{~g}$

Values are mean $+1 \mathrm{SD}$, in $\mathrm{mmol} / \mathrm{l}$

Temperature and air stability

Because the colonic environment is obviously anaerobic (Holdeman and Moore, 1972), and at $37^{\circ} \mathrm{C}$ it was considered relevant to study the effect of temperature and air on stool. Two experiments were performed in which diluted stool was divided and incubated at $37^{\circ} \mathrm{C}$ with either oxygen or nitrogen bubbling through. Results from one such experiment (Fig. 2), show that with oxygen there are considerable concentration increases of alanine, glutamate, glycine, lysine, and valine in $40000 \mathrm{~g}$ centrifugates, whereas under nitrogen there are virtually no changes. Other amino acid concentrations change in the same way but to a lesser extent, but taurine, urea, tryptophan, and histidine do not alter. In a given individual, the acids changing most on aerobic incubation were those which exhibited the greatest U-D difference. (The earliest measurement, recorded as $\mathbf{0}$ hours, is made at least three hours after sampling when allowances are made for handling and centrifugation time).

To see if temperature and air (as opposed to oxygen) were important in influencing fermentation or cell breakdown, diluted specimens were left in air, at room temperature or $4^{\circ} \mathrm{C}$ for 24 hours, after aliquots had been removed for base-line analysis. In all cases, there was a rise of groups III and IV amino acids (Fig. 3) in the oxygen containing environment, especially at warm temperatures. Under nitrogen, the situation is much more stable and no great changes were seen in the first nine hours at $37^{\circ} \mathrm{C}$.

\section{Discussion}

Our observations are devoted to the validation of high speed centrifugation and in vivo dialysis as

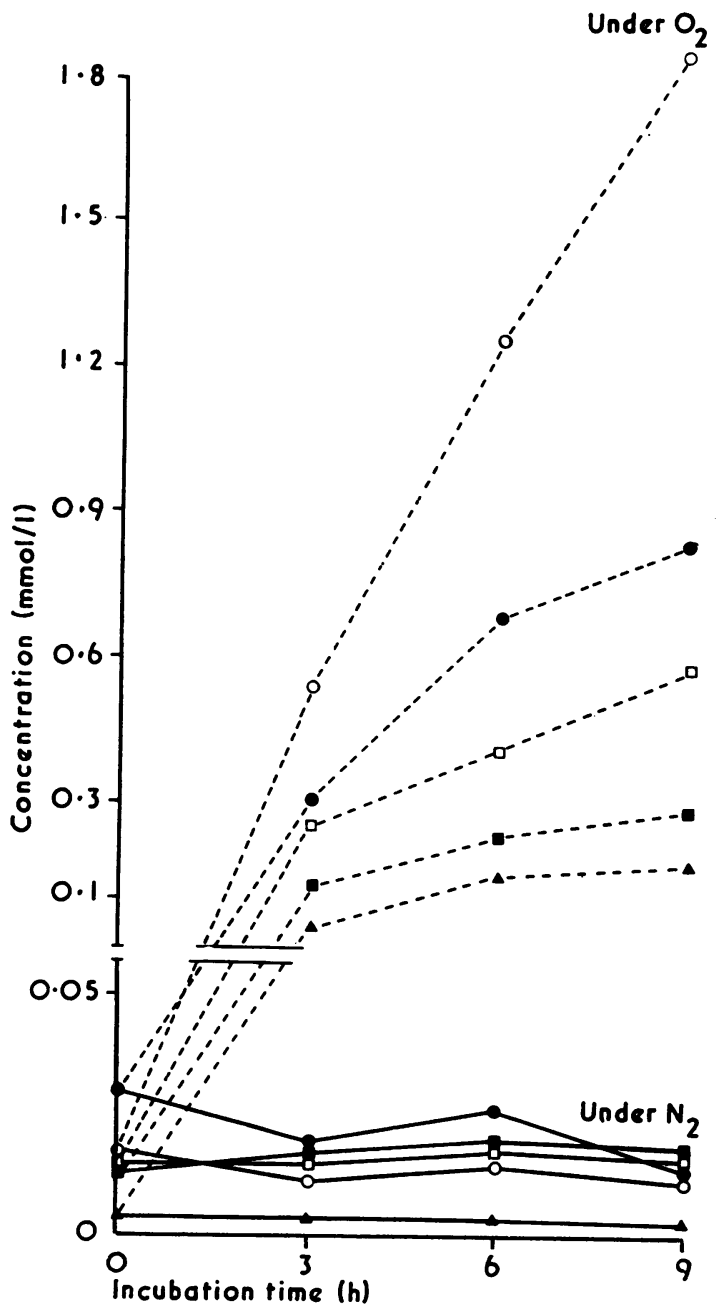

Fig. 2 Amino-acid concentration changes during incubation of diluted stool under nitrogen or oxygen at $37^{\circ} \mathrm{C}$ (○: Ala, O: Glu., $\square:$ Gly., $\square:$ Lys., $\Delta$ : Val.).

methods of sampling extracellular stool fluid. Very many physiochemical and biochemical factors affect the accuracy of these methods and we chose the amino acids as a group of substances that might help to delineate these various phenomena. They are easily and quantitatively estimated (Owens and Padovan, 1975), metabolically active, stable once isolated, and they exhibit a wide variety of physiochemical features such as size, shape, charge, hydrophylic and lipophilic properties. They are concentrated within living bacterial cells (Leach and Snell, 1960) and are therefore potentially intracellular markers.

Tarlow and Thom (1974) describe concentration 


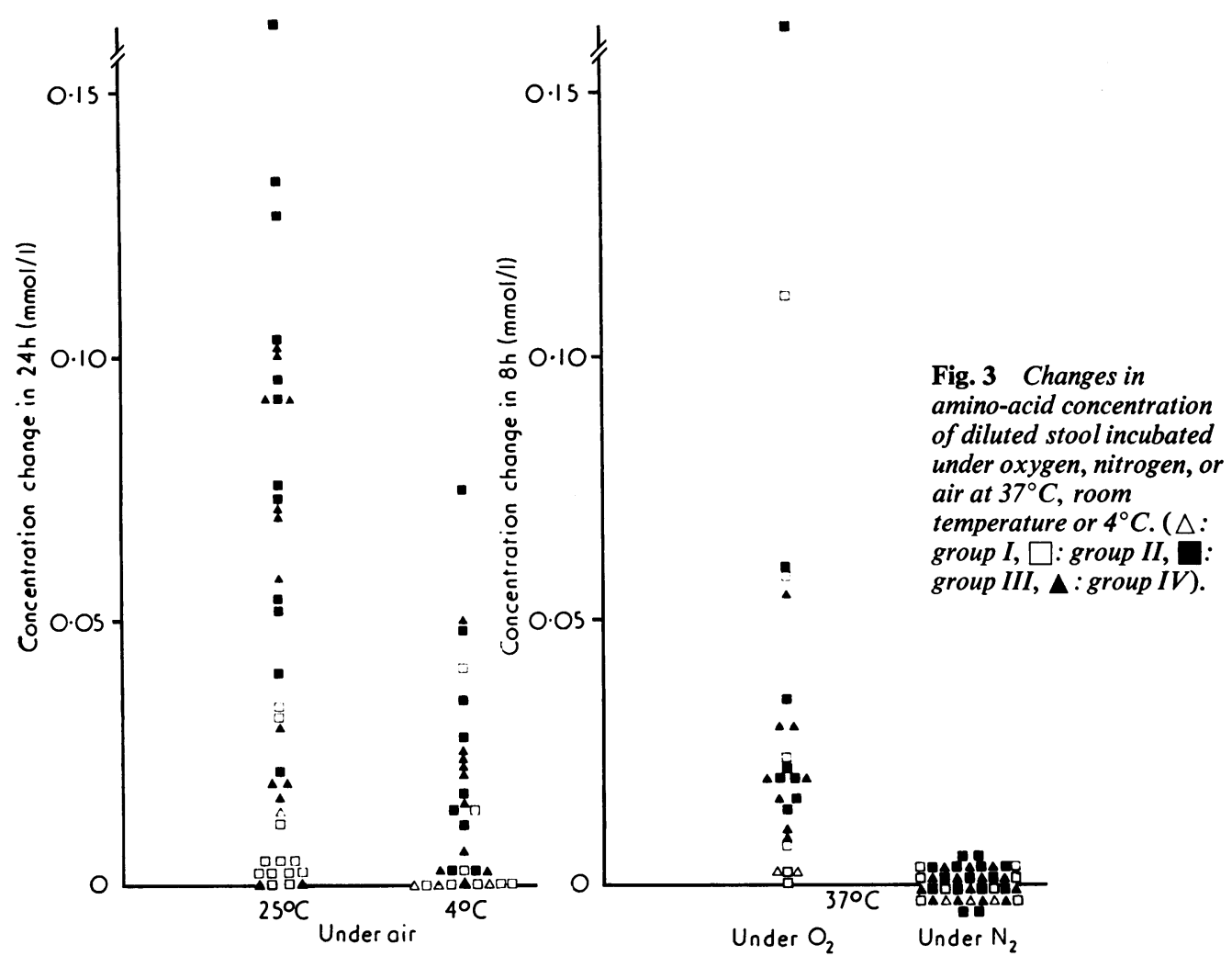

differences between ultracentrifugate and dialysate in titratable organic anion, sodium ion, ammonia, bicarbonate, total sugar and osmolality, with higher concentrations in the centrifugate except for bicarbonate. Titratable organic anions might include some ionised protein carboxyl groups which cannot diffuse into the sacks and therefore appear higher in centrifugate. We do not confirm their unexplained difference in sodium ion concentration. Ammonia status in the dialysate-centrifugate situation is complicated, since warm incubation in vitro leads to ammonia formation (Gamble, 1915). During sampling and centrifugation ammonia could be produced so leading to higher levels in the centrifugate, assuming that ammonia is generated by an enzyme system of molecular weight greater than 15000 (the exclusion size of the dialysis membrane) and, therefore, only occurs outside the bags. Similarly, the $\mathrm{pH}$ may fall secondary to continuing fermentation during centrifugation or storage and predispose even further towards carbon dioxide loss in the centrifuge head which is subject to a vacuum during operation, and so account for the lower levels of hydrogen ion and total $\mathrm{CO}_{2}$ seen in the centrifugate.

Higher concentrations of sugars and milliosmoles in centrifugate could also be produced by continuing fermentation and release of intracellular contents after cell death. We would like to present our data in this context. Table 1 shows that there are highly significant U-D differences for some amino acids and not others, which confirms a complex situation. Group I substances in Table 1 are estimated with difficulty: taurine is a poorly defined peak, urea is a poor colour reactor, glutamine is unstable on storage, and tryptophan shows variable binding. Significant differences could remain undetected, but it is unlikely. Group IV acids, however, are present in unequivocally higher concentrations after handling and centrifuging, but their origin is not absolutely certain. Aerobic incubation of an E. coli or Proteus modified the amino acid profile of the growth medium and produced a broth richer in many of the amino acids that show the greatest U-D difference and sonication of a saline suspension of a Proteus also produced a solution rich in group III and IV acids. These preliminary observations and the greater standard deviations for centrifugates are compatible with varying degrees of intracellular release and fermentation.

Sonication of diluted stool exaggerates the U-D differences for various amino acids (Fig. 1), while the data in Table 4 confirm that dilution in itself is 
not responsible. The gradient of the regression line depends on the intracellular concentration of free amino acids and the number of cells ruptured. The concentration of intracellular acids depends on the bacterial population present and therefore will be different in various individuals. Because of this, there is a close correlation between the amino acids liberated by centrifugation and sonication in a single individual, but accumulated data from several individuals had a much smaller but still significant correlation coefficient. We think individuals can be 'finger printed' into major groups with high lysine release, high glutamate and alanine release and so on.

Within the limitations of the methods used, there were no significant differences between the ionic concentrations of potassium, sodium or chloride in centrifugate and dialysate (Table 2). Sonication failed to increase the potassium concentration significantly, although a trend was seen. The intracellular potassium concentration of, for example, non-halidophile organisms (Brown, 1964) and E. coli (Cirillo, 1966) is only two to six times higher than the mean concentration of dialysate and is probably variable, depending on the state of growth and the time and temperature of storage. Failure to increase potassium concentration by sonication can be explained by lack of reproducibility in our analytical method and the similarity of the intra- and extracellular concentrations. Tarlow and Thom (1974) did not show loss of intracellular potassium either, despite freezing and storage at $0^{\circ} \mathrm{C}$ for 24 hours. Sodium and chloride concentrations in bacteria are similar to dialysate, hence major U-D differences or increases on sonication would not be expected and indeed were not found.

The aqueous diffusion data (Table 3) shows that the speeds of diffusion, in descending order, are Ure, Ser, Asp, Ala, Thr, Leu, His, Glu, Orn, Lys, whereas Table 1 shows quite a different order for the significance of the U-D difference (Ure $<\mathrm{His}<\mathrm{Ser}$ $<$ Glu $<$ Orn $<$ Thr $<$ Lys). Histidine with slow diffusion seldom displays a significant U-D difference, whereas alanine with moderately fast diffusion exhibits large differences. The U-D difference cannot therefore be explained by the diffusion characteristic of the dialysis bag.

In a stable aqueous based environment equilibrium is complete for most substances in two hours (Table 3), but molecular size, charge, $\mathrm{pH}$, and membrane characteristics are still important. The Table shows that stool centrifugate itself does not prevent equilibrium by modifying charges, creating chemical reactions, or by simply blocking membrane pores. The outer phase in this experiment was practically cell free (only a scanty growth of organism on culture) and was chemically stable.
Efficiency in any dialysis system depends on the speed of equilibrium across the membrane, the rate of concentration change in the surrounding medium and rates of diffusion through the medium to the dialysis surface. The experiments involving bags in diluted stool (Table 3) showed that alanine, valine, and isoleucine concentrations increased in the outside phase. They are all group IV amino acids and, in retrospect, it was clear that insufficient attention had been paid to the exclusion of air and that probably fermentation or intracellular release was proceeding faster than equilibrium was being achieved. Therefore, under rapidly changing conditions, there may well be limitations to the dialysis method. Under strictly anaerobic conditions (Fig. 2), amino acid concentrations are more stable. This was confirmed in a single experiment where one stool centrifugate and dialysate was prepared immediately and a second after incubation of the stool under nitrogen at $37^{\circ} \mathrm{C}$, for two hours. No marked differences were seen in either fluid as a result of time. We suggest that these anaerobic conditions approximate closely to the colon and, therefore, if faeces remain in the lower colon and rectum for more than two hours then in vivo analysis yields a representative fluid. This will probably be so for any person with one bowel action each day.

It seems likely that preparing a faecal centrifugate produces some artefacts, although simple anaerobic dilution $(1: 1)$ with saline and gentle homogenization appears relatively harmless. (A dilution ratio of $1: \frac{1}{2}$ faeces to saline is best avoided since it often produces an unexplained gel which prevents separation of a supernatent). Low speed $(6000 \mathrm{~g})$ supernatents have slightly higher aminoacid concentrations than their $26000 \mathrm{~g}$ (Table 5) or $40000 \mathrm{~g}$ (not shown) counterparts. The higher values in low speed preparations were attributed to cloudy supernatents containing cells that subsequently ruptured. The main differences were seen in glutamate and alanine, which is in accord with the concept of intracellular release (c.f. Fig. 1).

Figures 2 and 3 show that incubation under oxygen or air, as opposed to nitrogen causes release of especially group IV amino acids and that this can be slowed by reducing the temperature to $4^{\circ} \mathrm{C}$. The amino acids displaying insignificant $(P>0.01)$ U-D differences (open symbols) do not appear to be affected by these various parameters to the same extent. In a single experiment, incubation under oxygen at $4^{\circ} \mathrm{C}$ led to some increase in group IV amino acids but to a much lesser extent than at $37^{\circ} \mathrm{C}$. Incubation under nitrogen at $4^{\circ} \mathrm{C}$ was without changes and, in particular, did not suggest that there was leakage of amino acids from cells secondary to cooling alone. Whole stool exposed to air and kept 
at room temperature demonstrated obvious fermentation with gas formation and subsequent analysis again showed release of group IV amino acids. We take all these observations to suggest that exposure to oxygen or air results in anaerobe death and an increase in amino acids that are also released by sonication and which show the greatest U-D difference. It could be argued that these changes in amino acid concentrations are due to either the influence of a newly established aerobic population or the direct aerobic inhibition of enzymic conversions but in view of the speed of the changes we do not think this is likely. It does seem that, during normal laboratory handling procedures, stool suffers damage that modifies the validity of subsequent analysis.

We conclude that both methods of stool water sampling have limitations and that aerobically prepared ultracentrifugate is probably not equivalent to stool water. Under reasonably suitable conditions, dialysis probably provides the most convenient and accurate method of stool water sampling. If this is not possible for reasons of patient compliance, time, or rapid intestinal transit, then centrifugation is an alternative, providing the stool is kept under nitrogen, stored for a minimum period, preferably at $4{ }^{\circ} \mathrm{C}$ or room temperature, and centrifuged at $40000 \mathrm{~g}$.

Dr Padovan was supported by a scholarship from Fundacao de Amparo a Pasquisa do Estado de Șão Paulo (73/696), Brazil, and additional funds were provided by M.R.C. Grant No. G971/25/C. We are indebted to Dr D. C. Cusworth, Department of
Human Metabolism, for the facility of amino acid analysis and to Miss L. Lasley for technical assistance. Centrifugation and sonication was possible through the kind cooperation of Professor K. R. Rees, Department of Biochemical Pathology, and we are very grateful to $\mathrm{Mr} \mathrm{A}$. D. Lewis and Miss A. Shelldrake for providing us with electrolyte analyses in the Department of Chemical Pathology, University College Hospital. Finally, we thank Professor O. M. Wrong in whose department this work was done for his continued help and interest.

\section{References}

Brown, A. D. (1964). Aspects of bacterial response to ionic environment. Bacteriological Reviews, 28, 296-329.

Cirillo, V. P. (1966). Symposium on bioelectrochemistry of micro organisms. I. Membrane potential and permeability. Bacteriological Reviews, 30, 68-79.

Gamble, J. L. (1915). The ammonia and urea content of infants' stools with a description of methods. American Journal of Diseases in Childhood, 9, 519-532.

Holdeman, L. V., and Moore, W. E. C. (1972). Roll-tube techniques for anaerobic bacteria. American Journal of Clinical Nutrition, 25, 1314-1317.

Leach, F. R., and Snell, E. E. (1960). The absorption of glycine and alanine and their peptides by Lactobacillus casei. Journal of Biological Chemistry, 235, 3523-3531.

Owens, C. W. I., and Padovan, W. (1975). Quantitative method for estimating fecal amino acids. Clinical Chemistry 21, 1437-1440.

Tarlow, M. J., and Thom, H. (1974). A comparison of stool fluid and stool dialysate obtained in vivo. Gut, 15, 608-613.

Wrong, O. M., Metcalfe-Gibson, A., Morrison, R. B. I., $\mathrm{Ng}, \mathrm{S}$. T. and Howard, A. V. (1965). In vivo dialysis of faeces as a method of stool analysis. I. Technique and results in normal subjects. Clinical Science, 28, 357-375.

Wrong, O. M., Morrison, R. B. I., and Hurst, P. E. (1961). A method of obtaining faecal fluid by in-vivo dialysis. Lancet, 1, 1208-1209. 